\title{
Experimental study of a PEM Reversible Fuel Cell
}

\author{
S. RABIH, O. RALLIERES, C. TURPIN, S. ASTIER \\ University of Toulouse - Laboratory LAPLACE \\ Site ENSEEIHT - 2, rue Charles CAMICHEL \\ BP 7122, 31071 Toulouse Cedex 7, France \\ Tel: 33561588359 Fax: 33561638875 \\ name@laplace.univ-tlse.fr
}

\begin{abstract}
A Reversible (or Unitized) Fuel Cell is an electrochemical component which is able to operate by conception in two modes: in fuel cell mode (= production of electricity and heat starting from hydrogen and oxygen) as well as in electrolyser mode (production of hydrogen and oxygen starting from electricity and even from heat in certain cases).

Firstly, we present the operation principle and technological elements of a PEM RFC. Secondly, we study the quasi-steady behaviour of a PEM RFC through voltage-current curves. Thirdly, the dynamical behaviour is analyzed.
\end{abstract}

Keywords: reversible fuel cell, fuel cell mode, electrolyser mode, quasi static curve, dynamic behaviour.

\section{1- Introduction}

Fuel cells are studied through the world as means of generating electricity (and heat in certain cases) because of their high efficiency, quiet operation and environmental compatibility (if hydrogen is cleanly produced).

A fuel cell can be combined with a hydrogen generation device, commonly an electrolyser, to constitute a system often called "Regenerative Fuel Cell" (RFC). The confusion can exist with the Reversible Fuel Cell (RFC), also known as Unitized Regenerative Fuel Cell (URFC). We are using the initials URFC to avoid any confusion.

The URFC, object of this paper, is an only device capable of operating either in fuel cell mode or in electrolyser mode. The "fuel cell mode" means production of electricity (and heat) starting from hydrogen and oxygen; and the "electrolyser mode" means production of hydrogen and oxygen starting from electricity even from heat. Let us underline that the fluidic system around the URFC must authorize that reversibility, what can lead to more or less complex solutions. Nevertheless a URFC should offer reduced weight, volume, cost and complexity by combining the functions of fuel cell and electrolyser in the same hardware compared to a RFC system. A better dynamic response can be waited too.

Let us recall that, as early as 1839 , Sir William Grove discovered that it might be possible to generate electricity by reversing the electrolysis of water [1].

Current developments are achieved for aerospace and military applications. As an example, the American company Proton Energy Systems has been developing URFC technology since 1998. In [2], the authors modified a, commercial water PEM electrolyser, having an active area of $0.1 \mathrm{ft}^{2}$, to operate as a URFC...

This paper presents an experimental work about a PEM (Proton Exchange Membrane) reversible fuel cell. The tested PEM URFC is a commercially available low power cell which operates at atmospheric pressure. The following steps are presented in this paper:

- The operation principle of a PEM URFC system and some technological elements.

- The behaviour of the URFC system in quasisteady state.

- The switching between the two modes (fuel cell $\leftrightarrow$ electrolyser) under several shapes of currents.

\section{2- Operation principle and technological elements of a PEM URFC}

\section{2-1 Operation principle}

In the fuel cell mode, an URFC produces electricity from hydrogen and oxygen and generates heat and water as by-products according to the following reaction like any fuel cell:

$\mathrm{H}_{2}+\frac{1}{2} \mathrm{O}_{2} \rightarrow \mathrm{H}_{2} \mathrm{O}+$ electrical power + heat

In the electrolyser mode, an URFC absorbs electricity from a power source (solar, wind turbine...), heat from the system environment and/or from the losses of the system itself, to divide water into oxygen and hydrogen fuel according the follow reaction:

$$
\mathrm{H}_{2} \mathrm{O}+\text { electrical power }+ \text { heat } \rightarrow \mathrm{H}_{2}+\frac{1}{2} \mathrm{O}_{2}
$$

The total reaction of an URFC system is given by:

$\mathrm{H}_{2}+\frac{1}{2} \mathrm{O}_{2} \stackrel{\text { fielcellmode }}{\longrightarrow} \mathrm{H}_{2} \mathrm{O}+$ electrical power + Heat

The URFC system is drawn schematically in Fig 1. Let us underline that water here is an energy vector as well as hydrogen and oxygen: a water tank is required. The water tank is filled or emptied according to the operating mode like both gas 
tanks. The only exchanges with the environment of the URFC system are electrical power and heat. Such URFC system including the hydrogen, oxygen and water tanks is equivalent to an electrochemical battery: electricity can be stored or delivered at any time.

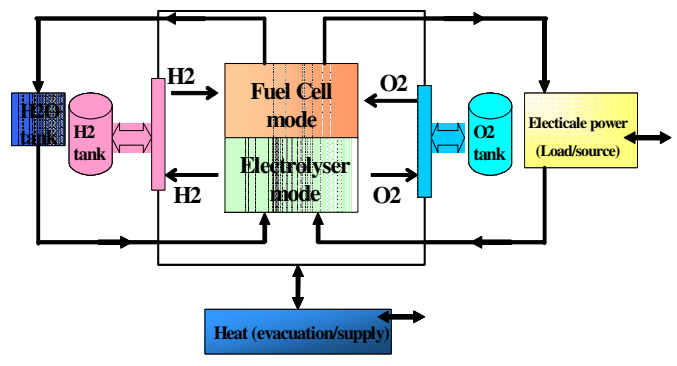

Fig. 1: Schematic operation of a reversible fuel cell system

\section{2-2 Technological elements}

An elementary PEM URFC consists of two electrodes (positive and negative) separated by a solid electrolyte. This electrolyte, typically in Nafion, is a membrane with a typical thickness of a few hundreds of micrometers; it must be a good electronic insulator and a good ionic conductor. Moreover this electrolyte is able to keep the oxygen and the hydrogen separated.

The electrode where hydrogen is consumed or produced is the negative electrode. The other one, where oxygen is consumed or produced, is the positive electrode. Most of works [3] deals with the positive electrode to make it possible the operating in both modes. Indeed, for example, for a classical PEM fuel cell, PTFE is added to facilitate the evacuation of the produced water; this PTFE has a negative effect in the electrolyser mode. A compromise has to be found as often.

Platinum is known as the most active catalyst for the electrochemical reaction at the negative electrode (hydrogen side) and $\mathrm{IrO}_{2}$ can be used at the positive electrode (oxygen side). [10]

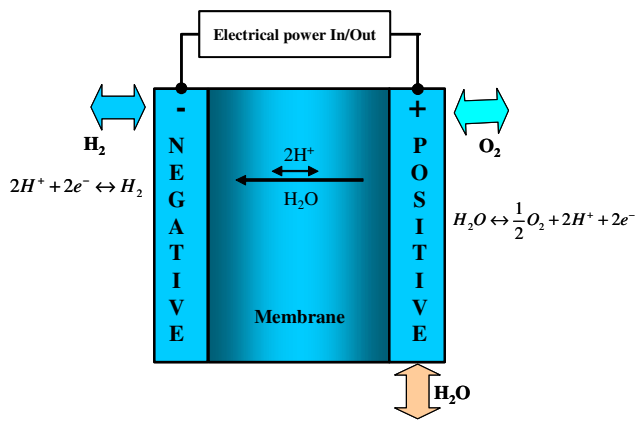

Fig. 2: Structure of an elementary PEM URFC

\section{2-3 Experimental set-up}

The used experimental set-up (fig. 3) is composed of the following elements:

- A commercially available elementary reversible PEM URFC with an activate area of $16 \mathrm{~cm}^{2}$ and a power of $2 \mathrm{~W}$ in electrolyser mode and of $0.6 \mathrm{~W}$ in fuel cell mode.

- A voltage generator which will supply the required power in electrolyser mode. Moreover, in both modes, this generator is required to assure the voltage conditions necessary for a good operating of the active load.

- An active load with high dynamic performances (large band-width) to impose the current on the URFC. Let us underline that the current is imposed in both modes, not the voltage.

- A current shunt to measure the current used for the regulation of the active load.

- A signal generator which gives the desired current reference.

- An insulated current sensor (Hall's effect) to measure the current flowing through the URFC.

- An oscilloscope to record the signals (voltage and current) of the URFC.

- A switching device which enables to change the direction of the current imposed to URFC according to the desired mode. Three positions are possible: stop, fuel cell mode and electrolyser mode.

Thank to the switching device, we can change instantaneously the operation mode with the same imposed current and observe the voltage behaviour of this elementary URFC.

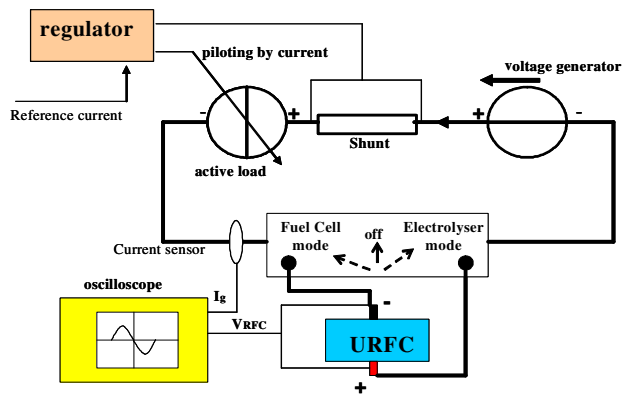

Fig. 3: Experimental set-up

The fluidic management allowing switching between both modes will be explained in the final paper.

\section{3- Behaviour of a PEM URFC in quasi- steady state}

We started by characterizing in quasi-steady state the URFC in the electrolyser mode. Indeed the fuel cell mode is not possible without gases. Then we inverted the current sign to characterize in quasisteady state the fuel cell mode. Then we 
characterized in quasi-steady state the URFC by an up-and-down sweeping of both modes.

The measurement of the URFC voltage is achieved directly at the terminals of the component, whereas the current is measured by an isolated current sensor.

\section{3-1 Voltage-current curve in quasi- steady} state in the electrolyser mode

The measurements were achieved at ambient temperature (around $20^{\circ} \mathrm{C}$ ) and atmospheric pressure. During the electrolyser operation, the two produced gases are stored in two separated tanks at pressures slightly higher than the atmospheric pressure.

Let us recall that the current was imposed and not the voltage. For each desired point, a new corresponding current reference was sent to the active load. After a non variation of the current during 30 seconds (stabilization of the current), we waited 3 minutes (stabilisation of the voltage) and took the operation point (measurements of voltage and current). Figure 4 shows the obtained quasistatic voltage-current curve of a PEM reversible cell in electrolyser mode $(2 \mathrm{~W})$.

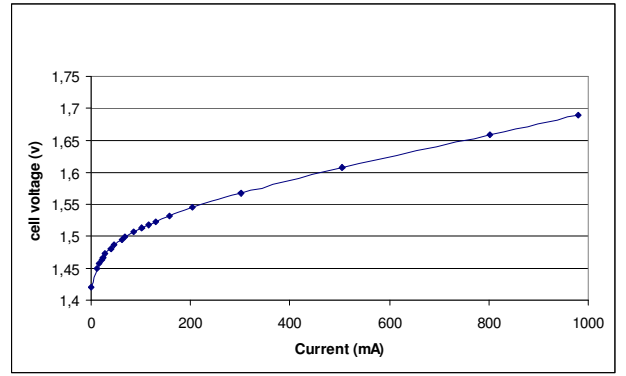

Fig. 4: Voltage-current curve in quasi-steady state, electrolyser mode

The shape of the obtained curve is typical. The measured open-circuit voltage at the temperature $20^{\circ} \mathrm{C}$ and atmospheric pressure is $E_{O C V \_E L}=1.42 \mathrm{~V}$.

This quasi-static curve results from the combination of all the following physicochemical phenomena: thermodynamic phenomena, activation phenomena, diffusion phenomena and ohmic losses. The static curve can be modelled by the following equation:

$U_{\text {cell_EL }}=E^{\text {rev }}+\eta_{\text {act_EL }}+R_{\Omega_{-} E L} I+R_{\text {diff_EL }} I$

$\mathrm{R}_{\Omega \text { EL }}$ models the ohmic losses (ionic conduction in the electrolyte, electronic conduction, and contact resistance) $\eta_{\text {act } E L}=\frac{R T}{n F} \operatorname{Ln}\left(\frac{I}{I_{0}}\right): \quad$ models the activation losses due to the kinetic phenomena. $R_{\text {diff_EL }}$ models the linear behaviour of diffusion losses in this current density range.

$E^{\text {rev }}$ is the reversible potential and is theoretically equal to $1.23 \mathrm{~V}$ [5].

Let us recall that the electrolysis occurs only when the cell receives energy equal to the reaction enthalpy $\Delta H$. Three cases can exist:
- $U_{\text {cell }}=\frac{\Delta H}{2 F}=1.48 \mathrm{~V} \Rightarrow \Delta Q_{e n v}=0$. There is no heat exchange with the environment. All the required energy is supplied by the voltage generator.

- $U_{\text {cell }}<\frac{\Delta H}{2 F} \Rightarrow \Delta Q_{e n v}<0$. The URFC absorbs heat from the environment (at maximum $\mathrm{T} \Delta \mathrm{S}$ ).

- $U_{\text {cell }}>\frac{\Delta H}{2 F} \Rightarrow \Delta Q_{e n v}>0$. The system gives heat to the environment because of the URFC losses. But the voltage generator must deliver the energy $\Delta \mathrm{H}$ and the losses of the URFC.

The measured open-circuit voltage $\mathrm{E}_{\mathrm{OCV}}$ is higher than the theoretical voltage $E^{r e v}$. An explication could be that we are in the case 2 thanks a parasitic micro/nano-current. In our tests, the cases 2 and 3 were very probably encountered.

The different parameters of the electrolyser mode are given in the table2.

\begin{tabular}{|c|c|c|c|}
\hline $\boldsymbol{\alpha}_{\text {EL }}$ & Io_EL & $\mathbf{R}_{\text {diff_EL }}$ & $\mathbf{R}_{\boldsymbol{\Omega} \mathbf{E L}}$ \\
\hline 0.74 & 2.51 & 93.4 & 96.6 \\
& $(\mathrm{~mA})$ & $(\mathrm{m} \Omega)$ & $(\mathrm{m} \Omega)$ \\
\hline
\end{tabular}

Table 2: Parameters of the electrolyser mode

\section{3-2 Voltage-current curve in quasi-steady state in the fuel cell mode}

The gases previously produced and stored in the two tanks were used to supply the URFC in the fuel cell mode $(0.6 \mathrm{~W})$. In this case, this URFC operates as an electrical generator. Compared to the previous case, the sign of the current is inverted but the polarity of the electrodes is unchanged. We plotted the quasi-static voltage-current curve under the same conditions as for the electrolyser mode (temperature, pressure and measurement time). Figure 5 shows the obtained quasi-static voltagecurrent curve of a PEM reversible cell in fuel cell mode.

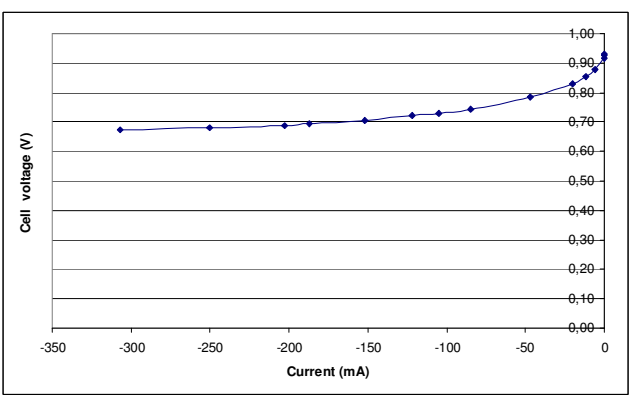

Fig. 5: Voltage-current curve in quasi-steady state, fuel cell mode

The shape of the obtained curve is quite classical even if the open-circuit voltage is quite low compared to the theoretical value $E^{\text {rev }}(1.23 \mathrm{~V})$ : $E_{O_{C V_{-} F C}}=0.932 \mathrm{~V}$ (at $20^{\circ} \mathrm{C}$ and atmospheric pressure). 
The physicochemical phenomena are the "same" in both modes: the "same" in terms of origins, but not quantitatively particularly for activation and diffusion losses. We can assume that they are identical for the ohmic losses. The static curve can then be modelled by the well-known following equation:

$U_{\text {cell_FC }}=E^{\text {rev }}-\eta_{\text {act_ } F C}-R_{\Omega_{-} F C} I-R_{\text {diff_ } F C} I$

The different parameters of the fuel cell mode are given in the table 1 .

\begin{tabular}{|c|c|c|c|}
\hline $\boldsymbol{\alpha}_{\text {FC }}$ & Io $_{\text {FFC }}$ & $\mathrm{R}_{\text {diff_FC }}$ & $\mathrm{R}_{\Omega \text { FC }}$ \\
\hline 0.702 & $4.17 e^{-8}$ & 237.6 & 102.3 \\
& $(m A)$ & $(m \Omega)$ & $(m \Omega)$ \\
\hline
\end{tabular}

Table (1): Parameters of the fuel cell mode

\section{3-3 Up-and-down voltage-current curve in quasi-steady state in both modes}

In both previous parts 3-1 and 3-2, we plotted the static curve independently for each operation mode under the same operating conditions (pressure, temperature and measurement time).

In this part, our aim is to plot the global static curve for the URFC for its two operation modes. For that, we began the measurements in the electrolyser mode to produce gases. The current was increased from 0 up to $950 \mathrm{~mA}$ ("up-scanning"). Then the current was decreased from $950 \mathrm{~mA}$ up to $-300 \mathrm{~mA}$ ("down-scanning"). Finally, the current was increased from -300mA up to 0 ("up-scanning"). The figure 6 gives the obtained up-and-down voltage-current curve. Let us precise that we waited only 1 minute for the stabilisation of the voltage (instead of 3 minutes previously).

We can note that, during the electrolyser operation:

- A hysteresis appears between the up and down points.

- The performances for the "up-scanning" are better; that means that it requires less energy to achieve the electrolysis. During the electrolyser mode, we note an increase of the gas bubbles with the increase of the imposed current. The electrolyser needs more energy to evacuate the gas bubbles (diffusion losses). When the current density increases, the membrane will be a priori less hydrated (water consumption); but the temperature increasing because of the loss increasing, the ionic conductivity increases compensating perhaps the previous effect. These two reasons could explain why the upscanning curve is better than the downscanning curve.

- The open-circuit voltage at the beginning of the "up scanning" $(1.40 \mathrm{~V})$ is lower than the one at the end of the "down-scanning" (1.435V).

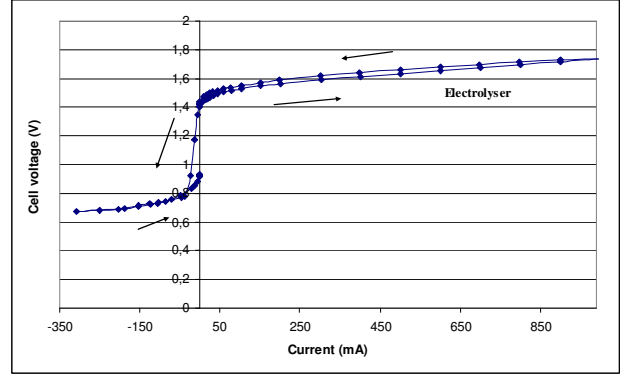

Fig. 6: Up-and-down voltage-current curve in quasi-steady state in both modes

On the other hand, during the fuel cell operation, we can notice that:

- The performances are worse than the in electrolyser mode. The operation is rather difficult to stabilize. This is probably due to the presence in excess of water due to the previous mode. Indeed, some water accumulated in the electrode pores and it results in electrode flooding, which degrades the cell performance by preventing the reactants from reaching the catalyst sites [6].

- The fuel cell mode occurs whereas the cell voltage is above $\mathrm{E}^{\mathrm{rev}}$. It is never the case for a classical fuel cell: the open-circuit voltage is always below $E^{\text {rev }}$ due to parasitical internal reactions. An explanation is that the mode switching instantaneously possible because the gases are present close to the catalyst site (they have been just produced!). The operation conditions of a classical fuel cell are progressively recovered as fluidic conditions change. It is confirmed by the behaviour during the up-scanning: the open-circuit voltage after the up-scanning is below $\mathrm{E}^{\mathrm{rev}}$.

- As soon as the classical operation in fuel cell is recovered, the up-scanning curve is better than the down-scanning. More energy is produced. This result is classical for a fuel cell.

\section{4- Behaviour of a PEM URFC vis-à-vis switchings of operation modes \\ 4-1 Dynamic response in voltage for switchings of operation modes}

Our aim was to observe the effect of quasiinstantaneous switchings between both operation modes on the URFC.

For that, we fixed the current at a certain value $\left|\mathrm{I}_{0}\right|$ for the two operation modes (electrolyser mode: $+\left|\mathrm{I}_{0}\right|$, fuel cell mode: $\left.-\left|\mathrm{I}_{0}\right|\right)$. We inverted the current sign manually thanks to our switching device. The switching time from one mode to the other one was here negligible. The switchings were forced every 40 seconds.

In order to observe the time evolution of the different quantities, we connected an oscilloscope to observe the cell voltage according to the sign of the forced cell current. Figure 7 shows the obtained 
results for currents of $\pm 200 \mathrm{~mA}$ and currents of $\pm 400 \mathrm{~mA}$. These values are naturally compatible with the current capabilities for both modes; the limitations being imposed by the fuel cell mode.

In Figure 7, it can be clearly observed that, each time a switching occurs, an instantaneous voltage drop (a few hundreds of $\mathrm{mV}$ ) occurs. It is due to the ohmic phenomena (mainly the electrolyte resistance) which react instantaneously to a current step.

After that instantaneous voltage variation, an "elbow" appears and is followed more or less rapidly by a linear variation of the cell voltage. If we make a zoom on this "elbow", it looks like a first-order response (exponential). For the authors, that corresponds to the charge (fuel cell mode $\Rightarrow$ electrolyser mode) or the discharge (electrolyser mode $\Rightarrow$ fuel cell mode) of the equivalent capacitor due to the double layer phenomena. This equivalent double layer capacitor makes it possible the instantaneous reversibility in current because of their electrostatic origin.

The linear variation of the cell voltage following the "elbow" has very probably a fluidic origin. Indeed, the fluidic phenomena can have a capacitive behaviour as demonstrated in [9] and observed in [8]. The charge (fuel cell mode $\Rightarrow$ electrolyser mode) and the discharge (electrolyser mode $\Rightarrow$ fuel cell mode) of this equivalent fluidic capacitor are an image of the switching of the fluidic behaviour due to the operation mode switching. This charge and discharge lasts several seconds to tens of seconds, leading to values of equivalent capacitances reaching several farads even more than ten farads. These values make it possible to reject, for the authors, the possibility of confusion between fluidic phenomena and double layer phenomena with regard to the cell area of $16 \mathrm{~cm}^{2}$; the equivalent double layer capacitance can be to a maximum of a few hundreds of millifarads.
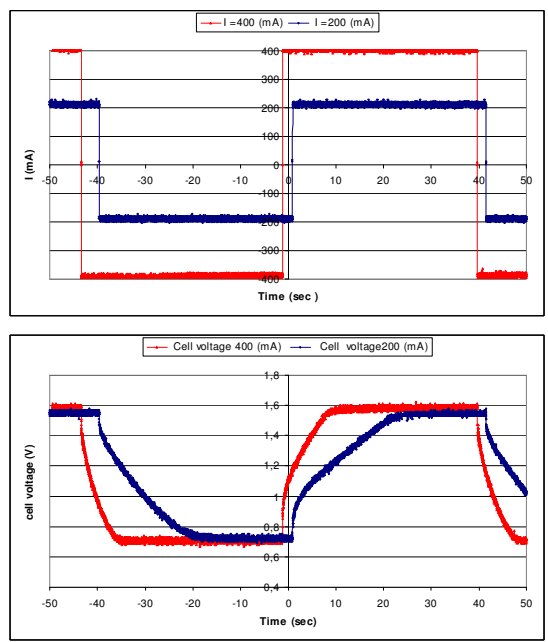

Fig. 7: Time evolution for switchings of operation mode every 40 seconds for $\mathrm{I}=200 \mathrm{~mA}$ and $\mathrm{I}=400 \mathrm{~mA}$
The following table shows the comparison of data for the different operation switchings for two values of current $200 \mathrm{~mA}$ and $400 \mathrm{~mA}$.

\begin{tabular}{|c|c|c|}
\hline Applied current (mA) & $\mathbf{2 0 0}$ & $\mathbf{4 0 0}$ \\
\hline $\begin{array}{c}\text { Duration of transient state } \\
\text { electrolyser mode } \Rightarrow \text { fuel cell mode } \\
\text { (seconds) }\end{array}$ & 25.5 & 6.5 \\
\hline $\begin{array}{c}\text { Duration of transient state } \\
\text { fuel cell mode } \Rightarrow \text { electrolyser mode } \\
\text { (seconds) }\end{array}$ & 28 & 8.5 \\
\hline $\begin{array}{c}\text { Duration of stabilized mode after the } \\
\text { switching electrolyser mode } \Rightarrow \text { fuel cell } \\
\text { mode (seconds) }\end{array}$ & 14.5 & 33.5 \\
\hline $\begin{array}{c}\text { Duration of stabilized mode after the } \\
\text { switching fuel cell mode } \Rightarrow \text { electrolyser } \\
\text { mode (seconds) }\end{array}$ & 12 & 31.5 \\
\hline $\begin{array}{c}\text { Instantaneous voltage drop after the } \\
\text { switching electrolyser mode } \Rightarrow \text { fuel cell } \\
\text { mode (mV) }\end{array}$ & 70 & 190 \\
\hline $\begin{array}{c}\text { Instantaneous voltage drop after the } \\
\text { switching fuel cell mode } \Rightarrow \text { electrolyser } \\
\text { mode (mV) }\end{array}$ & 120 & 180 \\
\hline
\end{tabular}

Table 1 Comparison of data for the different operation switchings for two values of current $200 \mathrm{~mA}$ and $400 \mathrm{~mA}$

From this table 1, we can notice that:

- The behaviour between the two operation modes is dissymmetrical even if the shapes of waves are similar.

- The duration of the transient state for the switching of the electrolyser mode towards the fuel cell mode is higher than for the switching of the fuel cell mode towards the electrolyser mode.

- The duration of the transient state is reduced with the increase of the imposed current. It is not proportional proving the existence of nonlinearities.

The influence of the fluidic phenomena is very important as already said. The switching of the electrolyser mode towards the fuel cell mode is the most constraining. Indeed some water accumulated within the pores of the positive electrode and constitutes a barrage to the flowing o the gases. But the measured differences are finally not so high.

\section{4-2 Introduction of pause times between each switching of operation modes}

In this part, we analyze the impact of the operation mode switchings on the open-circuit voltage. The shape of the imposed current is drawn in figure 8-a. Phases with a null current are introduced compared to previously. 


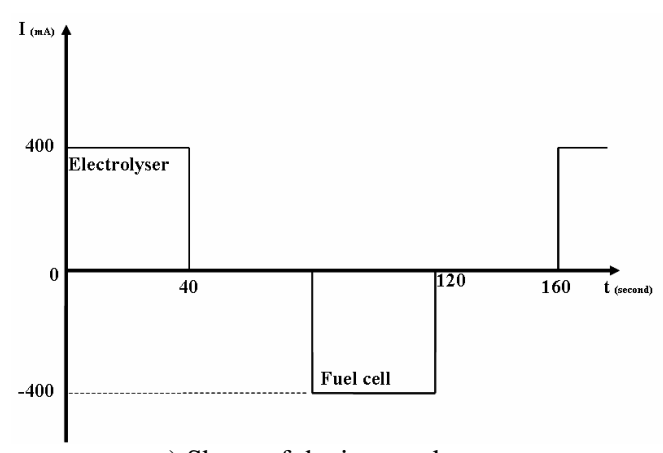

a) Shape of the imposed current

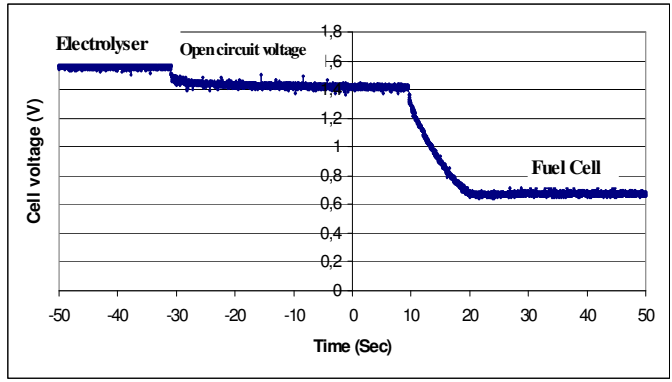

b) Electrolyser mode to fuel cell mode

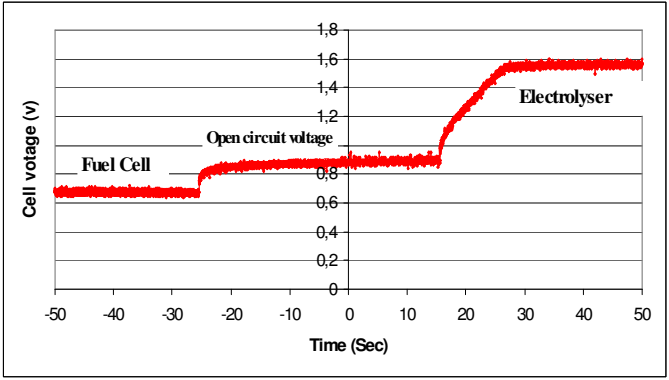

c) Fuel cell mode to electrolyser mode

Fig. 8: Time evolution with the introduction of pause times between the operation mode switchings

The figures 8-b and 8-c show the evolutions of the open-circuit voltage for the imposed current. We can divide the analysis into four steps:

1. A current step from $400 \mathrm{~mA}$ to $0 \mathrm{~mA}$ is applied (fig. 8-b). We observe the drop voltage due to the ohmic phenomena. Then an exponential decreasing can be observed due to the discharge of the equivalent double layer capacitor. The discharge duration under a null current confirms that its capacitance is limited to a few hundreds of millifarads. The opencircuit voltage tends towards $1.42 \mathrm{~V}$.

2. A current step from $0 \mathrm{~mA}$ to $-400 \mathrm{~mA}$ is applied (fig. 8-b). The cell here operates in the fuel cell mode. The evolution has the same shape as in part 4-1: instantaneous voltage drop (ohmic resistance), "elbow" corresponding to a firstorder evolution (discharge of the equivalent double layer capacitor), and linear decreasing (equivalent fluidic capacitor). The voltage finally tends towards its steady state.

3. A current step from $-400 \mathrm{~mA}$ to $0 \mathrm{~mA}$ is applied (fig. 8-c). It can be again observed the drop voltage due to the ohmic phenomena and the exponential increasing due to the charge of the equivalent double layer capacitor. The voltage increases until to reach the open-circuit voltage of $0.94 \mathrm{~V}$.

4. A current step from $0 \mathrm{~mA}$ to $400 \mathrm{~mA}$ is applied (fig. 8-c). The cell here operates in the electrolyser mode. The explanations are similar to the ones of the point 3 . The voltage finally tends towards its steady state $(1.6 \mathrm{~V})$.

This kind of tests demonstrates the existence of a time limitation due to fluidic phenomena for an operation mode switching, even if a pause time (null current). It also confirms the role of the double layer phenomena during transient states.

\section{5- Conclusion}

An experimental work was presented to investigate the behaviour of a commercially available reversible PEM fuel cell.

The quasi-steady state current-voltage curves plotted in each mode showed that the performances are not at all symmetrical. The up-and-down current-voltage curve plotted on the global operation area revealed hysteresic phenomena and a fluidic inertia at the operation mode switching.

The fluidic inertia was confirmed during all the tests of mode switchings through a capacitive behaviour of fluidic phenomena even if a pause time is applied between each mode.

This experimental work is currently used to establish an energy model of a reversible fuel cell. [9].

\section{6- References}

[1]- Mehrdad Ehsani, "Modern Electric, Hybrid Electric, and Fuel Cell Vehicles Fundamentals, Theory, and Design" Boca Raton London New York Washington, D.C. 2005 by CRC Press LLC. Ch1, PP 18.

[2]- Fred Mitlitsky, Blake Myers, Andrew H. Weisberg, Trent M. Molter and William F. Smith, "Reversible (unitised) PEM fuel cell devices", Fuel Cells Bulletin, Volume 2, Issue 11, August 1999, Pages 6-11.

[3]- Shidong Song, Huamin Zhang, Xiaoping Ma, Zhi-Gang Shao, Yining Zhang, Baolian Yi, "Bifunctional oxygen electrode with corrosion-resistive gas diffusion layer for unitized regenerative fuel cell", Electrochemistry Communications 8 (2006) 399-405.

[4]- W. Smith, "The role of fuel cells in energy storage", Journal of Power Sources 86_2000.74-83.

[5]- E. Rasten, "Electro catalysis in water electrolysis with solid polymer", Norwegian PhD thesis, October 2001.

[6]- N. Sammes, "Fuel Cell Technology Reaching Towards Commercialization", Springer PP: 108.

[7]- S. Busquet, C.E. Hubert, J. Labbé, D. Mayer, R. Metkemeijer , "A new approach to empirical electrical modelling of a fuel cell, an electrolyser or a regenerative fuel cell", Journal of Power Sources 134 (2004) 41-48.

[8]- W. Friede, «Modélisation et caractérisation d'une pile à combustible du type PEM », PhD thesis, INPL 2003.

[9]- R. Saisset, G. Fontes, C. Turpin, S. Astier, "Bond Graph Model of a PEM fuel cell". Journal of Power Sources, 156(1), pp. 100-107, mai 2006.

[10]- Shidong Song, Huamin Zhang, Xiaoping Ma, Zhi-Gang Shao, Yining Zhang, Baolian Yi, "Bifunctional oxygen electrode with corrosion-resistive gas diffusion layer for unitized regenerative fuel cell". Electrochemistry Communications 8 (2006) 399-405. 